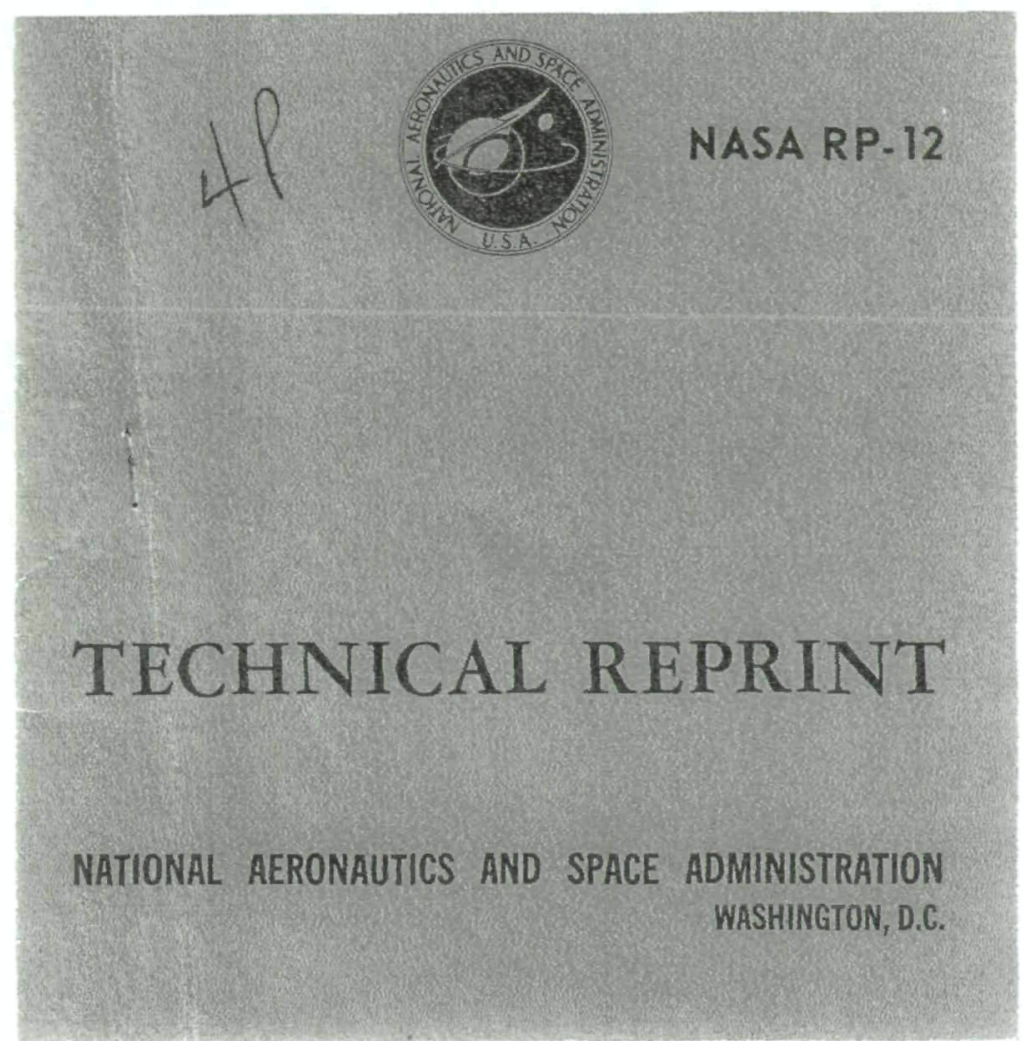




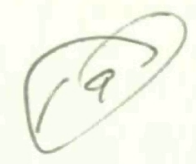

\section{N63 21388}

\section{Planets and Comets: \\ Role of Crystal Growth in Their Formation}

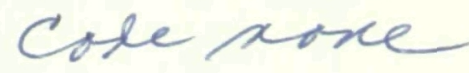

Bertram Donn

Gerald W. Sears 


\section{Planets and Comets: Role of}

\section{Crystal Growth in Their Formation}

21388 Abstract. The application of crystal growth theory to the formation of smoke particles from the primordial solar nebula indicates that solid particles with filamentary structures would form. Such particles would facilitate successive aggregation into planets, comets, and asteroids. The difficulties associated with the aggregation of spherical smoke particles would thus be avoided.

The earth and meteorites probably formed by accumulation of solid particles at temperatures below about $600^{\circ} \mathrm{C}(1,2)$. This concept has been accepted in nearly all recent attempts $(3,4)$ to make models of the synthesis of the solar system which would fit the requirements of current theories of stellar evolution. These studies suggest that sometime during the process, conditions favorable for the formation of small solid particles occurred. The fundamental problems are the detailed mechanisms of nucleation and growth of the primary particles and the mechanisms by which these particles aggregated to build larger objects. Several suggestions have been made concerning the latter: water and ammonia near the melting point by Urey $(2$, p. 122$)$, oily substances by Hoyle (5), and unspecified surface phenomena by Wood (6). These investigations assumed that spherical or equidimensional particles were present.

We consider the formation, structure, and properties of solid particles in the solar nebula according to crystal nucleation (7) and growth theory (8). The particles are expected to grow as filaments (9) and thin platelets (10) or combinations of both. These extremely nonspherical crystals with few crystal defects are referred to here as whiskers. We propose that these whiskers have properties that enable them to collect efficiently at first into loosely compacted aggregates resembling the lint balls that are often under beds, and then into larger objects.

Crystal formation from a supersaturated vapor phase occurs in two steps. The formation of the smallest stable volume of a crystal (about 30 molecules) is called nucleation. Subsequently, the crystal volume increases by the mechanisms of crystal growth.

Nucleation may occur either on a particle of a foreign solid (heteroge- neous) or in the vapor phase in the absence of foreign particles (homogeneous). The rate of nucleation is, in either case, a very sensitive function of supersaturation.

If the saturation vapor pressure of the bulk nucleating phase is $p_{0}$ and the partial pressure of the parent vapor phase is $p$, the supersaturation ratio $\alpha$ is given by

$$
\alpha=p / p_{0}
$$

and supersaturation, $\sigma$, is defined as

$$
\sigma=\alpha-1
$$

According to classical nucleation theory (7), the nucleation rate, $\mathrm{d} N / \mathrm{d} t$, nuclei created per cubic centimeter per second, for homogeneous nucleation is given by:

$$
\frac{\mathrm{d} N}{\mathrm{~d} t}=B \exp \quad\left(-\frac{A}{T^{3} \ln ^{2} \alpha}\right)
$$

For a given nucleation process, $A$ is constant and $B$ is approximately constant. $T$ is the absolute temperature.

The extreme sensitivity of nucleation rate on supersaturation or supercooling provides the characteristic feature of nucleation. There is a critical supersaturation, $\alpha^{*}$, below which nucleation does not occur at an appreciable rate and above which it occurs at a rapidly increasing rate. As nucleation occurs, the supersaturation is decreased and nucleation again ceases.

The growth of a nucleus occurs by the advance of the boundary surfaces. For a perfect crystal surface, continued growth requires the formation of new steps on the surface (11) by a process known as two-dimensional nucleation. A sensible growth rate usually requires a supersaturation greater than 50 percent for two-dimensional nucleation to occur.

A schematic plot of growth rate of a perfect surface from its own vapor is shown in Fig. 1A. Experimental curves, Fig. $1 B$, do not display the critical supersaturation characteristic of nucleation processes.

Frank (12) pointed out that actual crystals are not perfect but almost certainly contain screw dislocations. These are defects that provide growth steps of such a nature that the completion of a surface layer perpetuates rather than removes the defect as indicated by Fig. 2. This eliminates the need for the formation of new steps by two-dimensional nucleation and allows the crystal to grow at low supersaturations. Figure 3 shows the ap- pearance of the surface after several layers have been deposited upon it.

If a nucleus contains a single screw dislocation, it will grow at low supersaturations as a whisker $(9,13)$ whose axis coincides with the acting screw dislocation. If the nucleus contains a planar set of screw dislocations (14) it will grow as a very thin platelet. If it contains a three-dimensional array of screw dislocations, it will grow as an equiaxial crystal.

Since the presence of screw dislocations in growing crystallites allows equilibration of the supersaturated vapor phase with the stable crystal phase, the dislocations guarantee that the supersaturation rapidly diminishes after nucleation. Only those nuclei containing screw dislocations can grow, and the resultant smoke must contain whiskers or remain supersaturated at least to the degree required for two-dimensional nucleation. Growth at intermediate supersaturations occurs at certain reentrant twin boundaries.

Nuclei present in the pre-planetary nebula from the initial interstellar cloud are likely to lead to screw dislocations in the growing crystal $(9,14$, 15). Nuclei formed at high supersaturations also contain many defects (16)

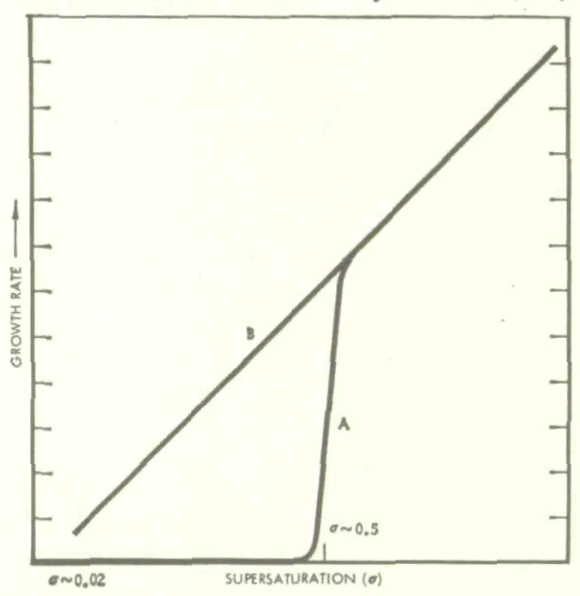

Fig. 1. (A) Theoretical curve for growth by two-dimensional nucleation. $(B)$ Typical experimental curve.

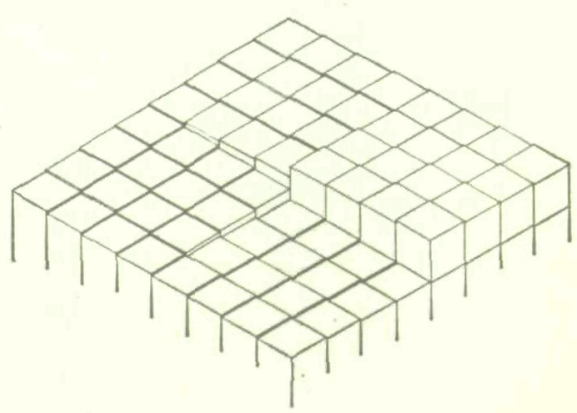

Fig. 2. Intersection of screw dislocation with crystal surface. The blocks represent molecules. 
and can grow as an array of whiskers (17). Radiation damage offers a possible source of screw dislocations which could offer a path to whisker formation.

We conclude that in the solar nebula a large fraction of the small crystals will be in the form of whiskers. Since perfect nuclei will be unable to continue growing in the presence of whiskers, the condensed solids on a mass basis will consist predominantly of whiskers.

Recent experiments at Cornell University (18) have shown that powders ground to $15 \mu$ diameter adhere readily on impact at velocities up to 15 $\mathrm{cm} / \mathrm{sec}$. They produce loosely packed structures that were called "fairy castles." These are very similar to our postulated lint balls.

The structure of filamentary particles would readily lead to coagulation by collision. The large ratio of surface to mass for whiskers compared to equiaxial particles further enhances the accumulation process. Any mechanism ' for forming aggregates of solid particles will be much more effective for whiskers than for spheres or equiaxed particles.

It is proposed that the whiskers collided to form lint balls. These balls provided a trap for spherical or equiaxed particles and also provided a tremendous internal area for absorption of noncondensable and slightly condensable gases.

Several properties of whiskers are of importance in the aggregation process.

1) Whiskers have strengths characteristic of perfect crystals. In general, they can be deformed 3 to 5 percent elastically (19). As an example, a whisker of pure iron (20) has withstood a tensile strain of 4.9 percent or a stress of $1.9 \times 10^{6} \mathrm{lb} /$ in. $^{2}$ For comparison, an ordinary iron crystal has an elastic limit of about $5000 \mathrm{lb} /$ in. $^{2}$ In bending, an elastic strain of 6 percent has been observed (21) compared with 0.01 percent elastic strain for ordinary pure iron.

2) Metal whiskers would not break into fragments upon mutual collision, but would, plastically, deform and intertwine. A lint ball of whiskers should be able to catch a relatively high-speed whisker or equiaxed particle.

3) A whisker has such a high ratio of surface to volume that any heat generated during a collision would be radiated away rapidly so that evaporative losses would be held to a minimum. A metal whisker $1 \mu$ in diameter mount- ed in a vacuum of $10^{-8} \mathrm{~mm}-\mathrm{Hg}$ has been observed to cool at $300^{\circ} \mathrm{C} / \mathrm{sec}$ from an initial temperature of $100^{\circ} \mathrm{C}$ at an ambient temperature of $25^{\circ} \mathrm{C}$ (21).

4) The large ratio of surface to volume would provide a large cross-section for whisker-whisker collisions as compared with collisions between equiaxed crystals by as much as a factor of 100 .

5) The junctures of whiskers and the dislocations generated during plastic flow associated with collision would create many internal sites suitable for further growth.

Throughout the solar nebula in the region now occupied by the planets, conditions were such that nonvolatile compounds such as silicates, some metals, and metallic oxides could condense. At some distance out in the nebula, temperatures were low enough for volatile substances, for example, water, ammonia, and hydrocarbons, to condense also (2). Shielding of solar radiation by the grains $(22,23)$ would have brought the zone of volatile condensation in much closer to the sun. If the shielding were sufficiently effective it may have permitted hydrogen condensation as well $(22,24)$.

A significant fraction of whatever substances did condense would have grown as whiskers.

Because of the high temperatures that were produced in the collapsing cloud $(2$, p. 122$)$ some of the condensed particles may have melted. Wood (6) has proposed that meteoritic chondrules were formed as liquid drops which then solidified or were possibly melted. Whatever processes did occur, in order to have the solid particles accrete into planets and meteorites, a majority of them must have formed as whiskers or on nuclei which grew as whiskers.

The time scale for accumulation of planets is an important consideration and requires careful analysis. Calculations have been carried out (25-27) for processes in which no mechanism of coagulation was given, but high efficiency of collection was assumed. In the

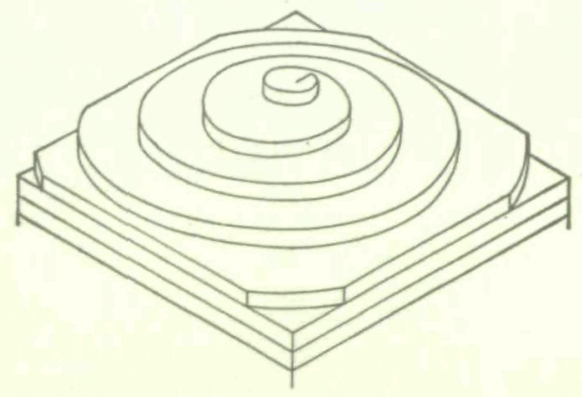

Fig. 3. Spiral growth at screw dislocation. calculation of Chandrasekhar (27) and von Weizsacker (26) a period of the order of $10^{8}$ years was obtained, if it is assumed that there was no significant depletion of cloud material. For a model in which the growing planets used up all the material, Schmidt obtained times of several billion years. Opik's studies (28) of collisions and accretion in interplanetary space are applicable and suggest times of $10^{6}$ to $10^{7}$ years (29).

A cluster of grains would have a very low density, perhaps about $0.1 \mathrm{~g} / \mathrm{cm}^{3}$ and possibly even lower, rather than densities of 3 to $8 \mathrm{~g} / \mathrm{cm}^{3}$ characteristic of stones or metals, which is usually assumed. Because of the low density, particles larger by a factor of about 5 than these calculated by Hoyle (3) would be carried along by the gas. This corresponds to a minimum diameter of $500 \mathrm{~cm}$.

Whatever the detailed processes, at some stage the "lint-ball" aggregates grew. Von Weizsacker, in his treatment of the problem (26), derived turbulent velocities of 3 to $10 \mathrm{~km} / \mathrm{sec}$ which were imparted to the small grains as well. The velocity must be limited to a value which would permit whiskers to coagulate. Iron whiskers could probably survive such impacts, silicates are much less likely to survive, and ice and other compounds of low boiling point would certainly not survive. A lower value seems required with a concomitant increase in time of accumulation. However, it appears that relative velocities for colliding smoke particles and small aggregates were much smaller.

The distance $\lambda$ within which the smoke velocity relative to the gas is reduced in the ratio $1 / e$ is given by $(27,30)$

$$
\lambda \approx \frac{\rho_{p}}{\rho_{o}} r
$$

$\rho_{p}$ is the particle density, $\rho o$ is the gas density, and $r$ is the effective radius. This must be multiplied by the ratio of particle velocity to thermal gas velocity when the ratio is less than unity. When in addition the particle diameter is greater than the mean free path which was presumably of the order of centimeters, Stokes law must be used. These different cases will not materially affect the conclusions. If we adopt $\rho_{p}=1$ $\mathrm{g} / \mathrm{cm}^{3}, \rho^{\prime}=10^{-12} \mathrm{~g} / \mathrm{cm}^{3}$ (3), and $r=$ $10^{-4} \mathrm{~cm}$, then $\lambda=1000 \mathrm{~km}$. For lintball aggregates with small $\rho_{p}, \lambda$ is still less.

The relative velocity of small aggregates was determined by the turbulent velocity of the gas within distances less 
than about $1000 \mathrm{~km}$. A very small relative smoke velocity could not have existed or the time of accumulation would have been excessively large.

The properties of whiskers considerably reduce the restrictions on the characteristics of the solar nebula in order for accumulation to occur.

As the aggregates grew, large relative velocities could develop between large aggregates and much smaller ones. Because of the porous, low-density structure of the large objects they could absorb the smaller ones with considerable efficiency, that is with little loss of material. Collisions between roughly equal size aggregates are much more difficult to analyze but considerable fragmentation is likely. A consequence of a high relative velocity between large and small aggregates would be a tendency for the larger objects to sweep up the smaller fairly rapidly and to facilitate growth.

Small nonvolatile coagulates which formed in or moved into regions where much volatile matter condensed would have been trapped in a porous, snowlike mass. Such combinations of volatile and nonvolatile low-density aggregates would account for Whipple's icy conglomerate comet nucleus (31). For this reason cometary grains are probably representative of the primordial condensate (32).

When solar heating vaporizes the ices of the comet nucleus, single whiskers, small fluffy aggregates, and trapped spherulites are released. Because of their large surface to mass ratio the more whiskery particles would be more readily carried along by the cometary gases and would also undergo greater repulsion by radiation pressure than the spherical or equiaxial particles. The difference between head and tail particles, to which Swings (33) called attention, may be primarily in the shape of the grains. This could be tested by observation (34).

Aggregates containing many grains which are released by the vaporization of the comet nucleus would spread out along the comet orbit. They would have the porous, fragile structure ascribed to dust ball meteors associated with comets (30, chap. 9; 35; 36).

Goddard Space Flight Center, National Aeronautics and Space Administration, Greenbelt, Maryland Gerald W. Sears

Molecular Physics Laboratory, General Dynamics, Electronics, Rochester, New York
References and Notes

1. H. Brown, in Atmospheres of the Earth and the Planets, G. G. Kuiper, Ed. (University of Chicago Press, Chicago, ed. 2, 1952); H. C. Urey, lecture, Intern, Congr. Pure Appl. Chem., 12th, Stockholm, 1953, p. 188; Appl. Year Book of Phys. Soc. Great Britain (1957), p. 14; - in Physics and Chemistry of the Earth, L. H. Ahrens, Ed. (Pergamon, New York, 1957), vol. 2; B. Y. Levin, Mem. Soc. Roy. Sci. Liège 18, 186 (1938).

2. H. E. Urey, The Planets (Yale University Press, New Haven, Conn., 1952).

3. F. Hoyle, Quart. J. Roy. Astron. Soc. 1, 28, (1960).

4. A. G. W. Cameron, Icarus 1, 13, (1962).

5. F. Hoyle, Frontiers of Astronomy (Heineman, London, 1955), chap. 6.

6. J. A. Wood, Geochim. Cosmochim. Acta 26, 739 (1962).

7. D. Turnbull, Solid State Physics (Academic Press, New York, 1958), vol. 3, p. 225.

8. W. K. Burton, N. Cabrera, F. C. Frank, Phil. Trans. Roy. Soc. London Ser. A 243, 299 (1951); C. Kittel, Introduction to Solid State Physics (Wiley, New York, ed. 2, 1953).

9. G. W. Sears, Acta Met. 1, 457 (1953),

10. J. B. Newkirk and G. W. Sears, ibid. 3, 110 (1955).

11. J. W. Gibbs, Collected Works (Longmans Green, London, 1928), p. 325.

12. F. C. Frank, Discussions Faraday Soc. 5, 48, 67 (1949).

13. G. W. Sears, Acta Met. 3, 361 (1955).

14. R. L. Fullman, J. C. Fisher, G. W. Sears, ibid. 2, 344 (1954).

15. J. B. Newkirk, ibid. 3, 121 (1955); M. I. Koslovski and M. V. Lomonosov, Krystallographia 3, 209 (1958).

16. G. W. Sears, J. Phys. Chem. 65, 1738 (1961).

17. J. E. Coleman, B. J. Allen, B. L. Allee, Science 131, 350, 1960.

18. B. Hapke and H. van Horn, Center for Radiophysics and Space Research, Cornell University, Report No. 139, in preparation.

19. S. S. Brenner, in Growth and Perfection of Crystals, R. H. Doremus, B. W. Roberts, D. Turnbull, Eds. (Wiley, New York, 1958), p. 157.

20. S. S. Brenner, Acta Met. 5, 131 (1957).

21. W. K. Murphy and G. W. Sears, unpublished results.

22. E. J. Opik, Icarus 1, 200 (1962).

23. B. Y. Levin, The Origin of the Earth and the Planets (Foreign Languages Publ. House, Moscow, ed. 2, 1958),

24. E. J. Opik, Mem. Soc. Roy. Sci. Liege, in press.

25. O. J. Schmidt, ibid. 15, 638 (1955).

26. C. F. von Weizsacker, Z. Astrophys. 22, 319 (1944).

27. S. Chandrasekhar, Rev. Mod. Phys, 18, 94 (1946).

28. E. J. Opik, Proc. Roy. Irish Acad. Sect. A 54, 165, (1951).

29. —, Astron. J. 66, 60, (1961).

30. - Physics of Meteor Flight in the Atmosphere (Interscience, New York, 1958), pp. 37-39.

31. F. L. Whipple, Astrophys. J. 111, 375 (1950).

32. B. Donn, Astron. J. 64, 126 (1959).

3. P. Swings, in Proc. Interdisciplinary Conf. on Electromagnetic Scattering, M. J. Kerker, Ed. (Pergamon, London, 1963).

34. B. Donn and R. S. Powell, ibid.

35. L. G. Jacchia, Astrophys. J. 121, 521 (1955).

36. The work of G. W. Sears was under contract NAS5-1590 between General Dynamics/Electronics/Rochester and the Goddard Space Flight Center. We thank Professor E. J. Opik for several valuable comments.

12 April 1963 